\title{
Projeto pedagógico do curso de Fisioterapia da Universidade de Brasília
}

\author{
Pedagogical plan for the physiotherapy program of the University of Brasilia
}

\author{
Vera Regina Fernandes Silva Marães ${ }^{[a]}$, Emerson Fachin Martins ${ }^{[b]}$, Gerson Cipriano Junior ${ }^{[\mathrm{c}]}$, \\ Ana Carolina Acevedo ${ }^{[\mathrm{d}]}$, Diana Lúcia Moura Pinho ${ }^{[\mathrm{e}]}$
}

[a] Doutora, professora da Faculdade de Ceilândia, Universidade de Brasília (UnB), Ceilândia, DF - Brasil, e-mail: veraregina@unb.br
[b] Doutor, professor da Faculdade de Ceilândia, Universidade de Brasília (UnB), Ceilândia, DF - Brasil, e-mail: efmartins@unb.br
[c] Doutor, professor da Faculdade de Ceilândia, Universidade de Brasília (UnB), Ceilândia, DF - Brasil, e-mail: cipriano@unb.br
[d] Doutora, professora da Universidade de Brasília (UnB), Brasília, DF - Brasil, e-mail: acevpoppe@gmail.com
[e] Doutora, professora e diretora da Faculdade de Ceilândia,Universidade deBrasília(UnB), Ceilândia,DF - Brasil, e-mail: diana@unb.br

\section{Resumo}

Introdução: A visível exaustão do modelo tradicional na formação do fisioterapeuta, a proposição de um novo perfil profissional e a mudança na abordagem no processo saúde-doença, que passa a observar a funcionalidade, vem estimular a necessidade de novos projetos pedagógicos para o ensino da fisioterapia. Este deve incluir uma perspectiva de transformação social e econômica da população regional, nesse caso o da região administrativa de Ceilândia, no Distrito Federal. Objetivo: Apresentar uma proposta de projeto pedagógico para curso de graduação em fisioterapia, valorizando a formação focada na funcionalidade humana em cenários de prática profissional propostos pelo Sistema Único Saúde (SUS). Resultados: O currículo está sendo construído numa perspectiva inovadora com propostas no âmbito geral (núcleos modo de vida, sistemas biológicos, instrumentalização em fisioterapia e cenários das práticas em fisioterapia); no âmbito específico (manutenção da saúde, processo saúdedoença, atenção à saúde, funcionalidade humana, processo permanente de formação); e no âmbito complementar (atividades de pesquisa e extensão). A proposta de projeto pedagógico foi pensada de forma a contemplar os objetivos gerais e específicos esperados, bem como dos perfis profissional e social. A indissociabilidade do ensino-pesquisa-extensão assume uma referência na relação professorestudante pela compreensão das atividades de pesquisa e extensão como elementos fundamentais do processo ensino-aprendizagem vinculado à vivência no contexto real, concretizando a relação teoria e prática, e com foco no SUS. Conclusão: A diversificação das práticas no contexto do SUS inclui uma perspectiva de transformação social e econômica da população no cenário social e político do Distrito Federal. Nesse contexto, prioriza-se a formação do profissional fisioterapeuta generalista, reflexivo e 
crítico, envolvido desde o princípio com o atendimento à comunidade nos diferentes níveis de atenção, voltado aos aspectos da funcionalidade humana.

Palavras-chave: Projeto pedagógico de curso. Fisioterapia. Ensino. Pesquisa. Extensão.

\begin{abstract}
Introduction: The visible exhaustion of the traditional teaching model for physiotherapy, along with a new professional profile and a change in the approach to the health-illness process (which has come to include functionality), has stimulated the need for new pedagogical projects for teaching physiotherapy. This should include a perspective of social and economic transformation of the regional population (in this case, the administrative region of Ceilândia, in the Federal District, Brasilia, Brazil). Objective: To present a pedagogical project for the undergraduate course in physiotherapy stressing education focused on human functionality in scenarios of professional practice,e proposed by the Brazilian public healthcare system. Methods: The curriculum is being constructed in an innovating perspective with proposals regarding the general realm (lifestyle centers, biological systems, physiotherapy skills and scenarios of physiotherapy practices), specific realm (health maintenance, health-illness process, healthcare, human functionality, process of permanenteducation) and complementary realm (research and extension activities). Results: The pedagogical project proposal was carried out in such a way as to complement the expected general and specific goals as well as professional and social profiles. The indissociability of teaching-research-extension presumes a reference in the professor-student relationship for the comprehension of research and extension activities as fundamental elements of the teaching-learning process linked to experience in the actual context, thereby concretizing the relationship between theory and practice, focusing on the public healthcare system. Conclusion: The diversification of practices in the context of the public healthcare system includes a perspective of social and economic transformation in the population within the social and political scenario of the Federal District. In this context, priority is given to the education/training of generalist, reflexive, critical physiotherapists, involved from the beginning with community service on different levels of healthcare, addressing aspects of human functionality in accordance with the needs of the Brazilian public healthcare system.
\end{abstract}

Keywords: Pedagogical course plan. Physiotherapy. Teaching. Research. Extension.

\title{
Introdução
}

A Fisioterapia surgiu como mais um elemento no processo de reabilitação das condições incapacitantes, especialmente em consequência de momentos históricos como a Revolução Industrial e as Grandes Guerras Mundiais, nos séculos XVIII e XX (1). No Brasil, a regulamentação da profissão em nível superior ocorreu pelo Decreto Lei n. 938 de 13 de outubro de 1969 (2), em cujo Artigo $3^{\circ}$ atribui-se ao profissional a execução de métodos e técnicas fisioterapêuticas, com a finalidade de restaurar, desenvolver e conservar a capacidade física do paciente (3).

Como profissão de saúde, deve-se recordar que nesta época o País se encontrava em pleno regime militar, no início dos diálogos relativos à reforma sanitária, e que na década de 1970 se discutia a necessidade de implementar políticas públicas mais efetivas e democratizadas em saúde. Esse movimento, responsável pelas Conferências Nacionais de Saúde, realizadas nas décadas de 1970 e 1980, mas que somente após o fim da ditadura, em 1990, consegue criar o Sistema Único de Saúde (SUS) nos moldes que hoje conhecemos (4).

Neste contexto, a formação do fisioterapeuta, que até o presente momento vinha sendo realizada por herança do modelo médico, baseada na criação das especialidades e formando fisioterapeutas para tratar doenças e processos incapacitantes em partes do corpo, passa a questionar sua atuação e participação nos âmbitos de atenção à saúde, de acordo com o modelo proposto pelo SUS $(1,3)$. 
Mais tarde, o avanço no processo de globalização viria favorecer a interlocução e discussão do papel do profissional fisioterapeuta, definido em 1999, pela Confederação Mundial de Fisioterapia (WCPT), como profissional que presta serviços a pessoas e populações, com o fim de desenvolver, manter e restaurar o movimento e a capacidade funcional em todos os ciclos de vida, no contexto da promoção, prevenção, tratamento e reabilitação (5).

Mais recentemente, a visível exaustão do modelo tradicional de formação acadêmica, a pequena inserção desse profissional no Sistema de Saúde, a proposição de um novo perfil profissional a partir das Diretrizes Curriculares Nacionais (DCN) do Curso de Graduação em Fisioterapia, e a mudança na abordagem no processo saúde-doença, que passa a observar a funcionalidade, de acordo com a proposição da Classificação Internacional da Funcionalidade (CIF), proposta pela Organização Mundial em Saúde (OMS) em 2002, tudo isso vem estimular de forma séria e profunda os projetos pedagógicos para o ensino da Fisioterapia nos tempos atuais $(6,7)$.

A formação do fisioterapeuta, na graduação, com um direcionamento para a funcionalidade humana por meio de intervenções norteadas pelos níveis de complexidade do SUS, como forma de mudança nos paradigmas atuais, é a cada dia mais eminente e necessária, a fim de contribuir com a formação enquanto profissional de saúde, o que certamente culminará em resultados favoráveis à qualidade e inserção no mercado de trabalho, identificando melhor as ações do fazer em fisioterapia (8-11).

Tal reforma tem como ator principal no processo as Universidades Públicas, que além de estarem historicamente comprometidas com o ensino e a reforma sanitária brasileira, têm recebido atualmente apoio e incentivo do Governo Federal, a partir da proposta nacional de Renovação e Expansão Universitária (REUNI) do Ministério da Educação (MEC), fornecendo subsídios para essas mudanças por meio da criação de novos cursos de graduação e, consequentemente, do aumento do número de vagas públicas em curso de graduação em Saúde - cursos estes que também devem ter forte interlocução e apoio com as iniciativas do Ministério da Saúde.

Um curso de graduação em Fisioterapia nos dias atuais deve oferecer ao futuro profissional uma visão crítica e problematizadora da natureza social do processo saúde-doença, sem deixar de contemplar a formação técnica e científica, que deve estar expressa tanto na sua estrutura curricular quanto em sua opção metodológica. Outro desafio é romper com os modelos disciplinares rígidos na busca do aperfeiçoamento da formação do fisioterapeuta, numa integração de diferentes conhecimentos, áreas disciplinares e profissionais (7, 8, 12-14).

Dessa forma, o objetivo deste estudo é apresentar uma proposta de projeto pedagógico inovadora para curso de graduação em Fisioterapia, valorizando a formação voltada para a funcionalidade humana em cenários de prática profissional que atenda ao modelo de atenção à saúde proposto pelo SUS, contribuindo com a evolução e melhor contextualização do fisioterapeuta enquanto profissional de saúde.

\section{Método}

A proposta pedagógica do curso de Fisioterapia nasce de um projeto construído coletivamente, concebido com base numa concepção de educação que compreende o sujeito aprendiz como pleno de possibilidades, e que não se limita a uma função meramente instrumental. Uma educação que não se coloca a serviço da reprodução ou da mera transmissão de informações, valores e crenças que imobilizam sujeitos e coletividades. Nesse sentido, o projeto pedagógico tem como foco o sujeito aprendiz e busca assegurar ao graduando uma formação integral, que mantenha uma relação orgânica entre ensino, pesquisa e extensão. Utiliza as DCN, mantendo dessa forma a coerência e legalidade da proposta (7). Busca uma estrutura curricular de caráter inovador, ao mesmo tempo em que se vincula e reforça a consolidação do SUS. Propõe a integração entre as áreas Biológicas, Humanas e Sociais, Ciências Exatas, Saúde e áreas profissionalizantes específicas, incluindo uma perspectiva de transformação social e econômica da população regional, no caso da região administrativa de Ceilândia, no Distrito Federal.

A proposta concentra-se em soluções eficientes e inovadoras, as quais serão aqui detalhadas como âmbito geral, descrevendo aspectos considerados no Projeto Político Pedagógico Institucional da Faculdade 
de Ceilândia, no que se refere às diretrizes balizadoras para todos os cursos ofertados; complementar, descrevendo estratégias andragógicas de aprendizagem (15); e específico, descrevendo aspectos considerados no Projeto Pedagógico do Curso de Fisioterapia $(16,17)$.

No âmbito geral, a estrutura curricular está organizada de modo a assegurar aos discentes o contato e experiência em conteúdos, abordagens e situações práticas de articulação no ensino e nos serviços, de maneira integrada, distribuídos em estruturas interdependentes, denominadas eixos estruturantes, que traduzem os núcleos de formação. São eles:

1) Núcleo Modo de Vida: apresenta um predomínio de conteúdos e abordagens voltadas à aproximação dos estudantes com o processo de trabalho enquanto produção e reprodução da sociedade em âmbitos local, regional e geral; as relações sociais que lhes são características e campo de saberes e práticas em saúde que lhes são inerentes;

2) Núcleo de Sistemas Biológicos: desenvolve-se privilegiando conteúdos e abordagens voltadas à compreensão dos sistemas biológicos que podem ser afetados durante o processo saúde-doença;

3) Núcleo Instrumentalização em Fisioterapia: enfatiza aqueles aspectos, conteúdos e abordagens, voltados à qualificação da ação dos estudantes nas questões diretamente implicadas na aplicação técnica dos recursos fisioterapêuticos;

4) Núcleo Cenários das Práticas em Fisioterapia: destaca conteúdos, abordagens e vivências voltadas ao exercício da reflexão e proposição de práticas fisioterapêuticas (Figura 1).

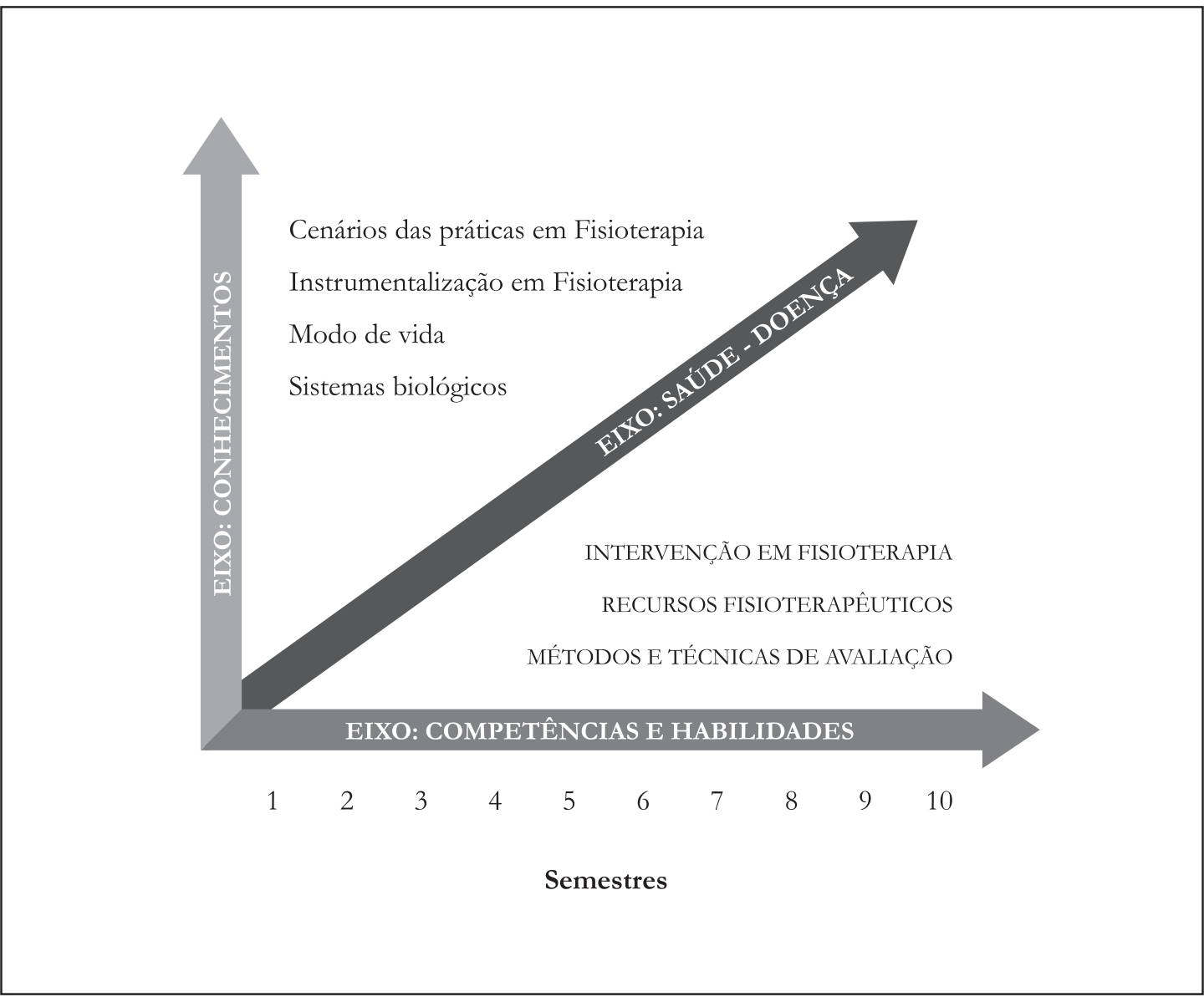

Figura 1 - Desenho curricular do curso de Fisioterapia apresentando os eixos estruturantes, módulos e conteúdos 
Nesses dois últimos Núcleos encontram-se os módulos em que serão discutidos conteúdos por meio de blocos temáticos, que estão relacionados às áreas de conhecimentos biotecnológicos e fisioterapêuticos previstas nas DCN do curso de Fisioterapia. Embora cada núcleo concentre, por razões didáticas, um conjunto de conteúdos e abordagens próprias, ao longo do curso o corpo discente terá a oportunidade de entrar em contato com elementos dos quatro eixos estruturantes, ainda que com graus de profundidade distintos.

No âmbito específico, o projeto pedagógico de curso de graduação em Fisioterapia está estruturado para responder às necessidades de formação, em graduação, de profissional em saúde capacitado a atuar em todos os âmbitos de sua atenção, com visão ampla e global da funcionalidade humana, em todas as suas formas de expressão individual e coletiva, por meio de recursos terapêuticos físicos e manuais, próprios de seus atos privativos (12). Para tanto, orienta-se por metodologias ativas e emancipadoras, que têm como eixo principal a construção das competências e habilidades que valorizem o significado da experiência do discente e a sua individualidade. No âmbito específico, o projeto pedagógico fundamenta-se em alguns enfoques, a saber:

a) manutenção da saúde: entendida em um enfoque ampliado, incorporando a visão subjetiva dos sujeitos e sua percepção sobre saúde e qualidade de vida;

b) processo saúde-doença: entende a saúde e doença como processo, oriundo não só dos fenômenos biológicos, mas também resultante do modo como os seres humanos estabelecem relações com o meio e entre si, enquanto indivíduos ou grupos, um processo que compreende os fatores de vida, de adoecimento e de morte dos seres humanos, permeando o ciclo vital, envolvendo as dimensões espirituais, relacionais e éticas, em um contexto socioeconômico e cultural;

c) atenção à saúde: tem com base os princípios do SUS, que supõe a integralidade das ações, o trabalho em equipe multiprofissional e transdisciplinar, centrada em atitudes ética e política, em sintonia com a co-responsabilidade, intersetorialidade, reconhecendo os limites do conhecimento e das tecnologias, possibilitando a construção de relações contínuas como forma de responder às necessidades sociais em qualidade de vida e saúde;

d) funcionalidade humana, entendida pelas perspectivas do corpo, do indivíduo e da sociedade o termo funcionalidade humana é então definido pelas estruturas e funções corporais, pelas atividades humanas e pela participação em nível individual e coletivo que se contrapõe ao termo deficiência que caracteriza pela limitação às atividades humanas e a restrição à participação individual e coletiva em um modelo de funcionalidade, incapacidade e saúde;

e) processo permanente de formação: considerando que a graduação, com a sua terminalidade, é uma etapa do processo da formação que continua ao longo da vida profissional do indivíduo, este projeto possibilita a continuidade do processo de formação, por meio da pós-graduação e da educação permanente, que valorizam a gestão do conhecimento como uma atividade que compõe o cotidiano das atividades da rede de serviços $(18,19)$.

Finalmente, no âmbito complementar, estão previstas atividades de pesquisa e de extensão que oferecerão possibilidades adicionais de ensino e contato profissional durante toda a formação, cujos conteúdos estão dispostos não necessariamente de maneira linear e progressiva. Da mesma forma, as atividades teóricas e práticas da formação específica em Fisioterapia serão desenvolvidas gradualmente desde o início do curso.

\section{Resultados e discussão}

Como produto das concepções apresentadas, o curso de Fisioterapia foi estruturado conforme apresentado na Tabela 1. 
Tabela 1 - Organização em Núcleos, Módulos e Blocos Temáticos dos conteúdos obrigatórios

\begin{tabular}{|c|c|c|}
\hline \multicolumn{3}{|c|}{ Núcleo Modo de vida } \\
\hline Módulos & Blocos temáticos & Semestre \\
\hline \multirow[t]{2}{*}{ Comunicação e Expressão } & Português Instrumental & $1^{\circ}$ \\
\hline & Metodologia Científica & $3^{\circ}$ \\
\hline \multirow[t]{2}{*}{ Saúde e Sociedade } & Saúde e Sociedade: Ciências Sociais & $1^{\circ}$ \\
\hline & Saúde e Sociedade: Relações em Saúde & $2^{\circ}$ \\
\hline \multirow[t]{2}{*}{ Epidemiologia } & Epidemiologia Descritiva & $1^{\circ}$ \\
\hline & Epidemiologia Analítica & $2^{\circ}$ \\
\hline \multirow[t]{2}{*}{ Políticas de Saúde } & Modelos e Práticas de Atenção à Saúde & $3^{\circ}$ \\
\hline & Políticas e Sistemas de Serviços em Saúde & $4^{\circ}$ \\
\hline \multicolumn{3}{|c|}{ Núcleo de Sistemas Biológicos } \\
\hline Módulos & Blocos temáticos & Semestre \\
\hline \multirow[t]{2}{*}{ Do Átomo à Vida } & Fundamentos Químicos e Biológicos & $1^{\circ}$ e $2^{\circ}$ \\
\hline & Desenvolvimento e Metabolismo & $3^{\circ}$ e $4^{\circ}$ \\
\hline \multirow[t]{4}{*}{ Das Células aos Sistemas } & $\begin{array}{l}\text { Organização dos sistemas e sistema } \\
\text { musculoesquelético e articular }\end{array}$ & $1^{\circ}$ \\
\hline & Sistema nervoso & $2^{\circ}$ \\
\hline & Circulatório, Respiratório e Digestório & $3^{\circ}$ \\
\hline & Revestimento Corporal, Ginecológico e Urinário & $4^{\circ}$ \\
\hline \multirow[t]{2}{*}{ Mecanismos de Agressão de Defesa } & $\begin{array}{l}\text { Agressão, Lesão, Adaptação, Defesa, Reparo, } \\
\text { Recuperação e Morte }\end{array}$ & $3^{\circ}$ \\
\hline & Agressão Microbiológica, Parasitárias e Defesa & $4^{\circ}$ \\
\hline \multicolumn{3}{|c|}{ Instrumentalização em Fisioterapia } \\
\hline Módulos & Blocos temáticos & Semestre \\
\hline \multirow[t]{4}{*}{ Fundamentos de Fisioterapia } & Generalidades & $1^{\circ}$ \\
\hline & Agentes Físicos & $2^{\circ}$ \\
\hline & Movimento & $3^{\circ}$ \\
\hline & Trofismo e Plasticidade & $4^{\circ}$ \\
\hline
\end{tabular}


Tabela 1 - Organização em Núcleos, Módulos e Blocos Temáticos dos conteúdos obrigatórios

(conclusão)

\begin{tabular}{|c|c|c|}
\hline \multicolumn{3}{|c|}{ Instrumentalização em Fisioterapia } \\
\hline Módulos & Blocos temáticos & Semestre \\
\hline \multirow[t]{3}{*}{ Fisioterapia Baseada em Evidências } & $\begin{array}{l}\text { Evidências de Métodos e Técnicas de Avaliação da } \\
\text { Funcionalidade }\end{array}$ & $5^{\circ}$ \\
\hline & $\begin{array}{l}\text { Evidências de Aplicação dos Recursos } \\
\text { Fisioterapêuticos }\end{array}$ & $6^{\circ}$ \\
\hline & Evidências de Intervenção Fisioterapêutica & $7^{\circ}$ \\
\hline $\begin{array}{l}\text { Gestão de Serviços e Recursos Humanos em } \\
\text { Fisioterapia }\end{array}$ & Bloco Temático Único & $6^{\circ}$ \\
\hline Seminários Integradores & Atenção Básica e de Média e Alta Complexidade & $9^{\circ}$ \\
\hline \multicolumn{3}{|c|}{ Núcleo de Cenários de prática profissional em Fisioterapia } \\
\hline Módulos & Blocos temáticos & Semestre \\
\hline \multirow[t]{3}{*}{ Fisioterapia na Atenção Básica } & Métodos e Técnicas de Avaliação & $5^{\circ}$ \\
\hline & Aplicação dos Recursos Fisioterapêuticos & $6^{\circ}$ \\
\hline & Intervenção em Fisioterapia & $7^{\circ}$ \\
\hline \multirow[t]{3}{*}{ Fisioterapia na Atenção de Média Complexidade } & Métodos e Técnicas de Avaliação & $5^{\circ}$ \\
\hline & Aplicação dos Recursos Fisioterapêuticos & $6^{\circ}$ \\
\hline & Intervenção em Fisioterapia & $7^{\circ}$ \\
\hline \multirow[t]{3}{*}{ Fisioterapia na Atenção de Alta Complexidade } & Métodos e Técnicas de Avaliação & $5^{\circ}$ \\
\hline & Aplicação dos Recursos Fisioterapêuticos & $6^{\circ}$ \\
\hline & Intervenção em Fisioterapia & $7^{\circ}$ \\
\hline \multirow[t]{3}{*}{ Fisioterapia no Processo de Reabilitação } & Métodos e Técnicas de Avaliação & $8^{\circ}$ \\
\hline & Aplicação dos Recursos Fisioterapêuticos & $8^{\circ}$ \\
\hline & Intervenção em Fisioterapia & $8^{\circ}$ \\
\hline \multirow[t]{2}{*}{ Estágio Profissional em Fisioterapia } & Atenção Básica e de Média Complexidade & $9^{\circ}$ \\
\hline & Atenção de Alta Complexidade & $10^{\circ}$ \\
\hline
\end{tabular}

A avaliação da proposta de projeto pedagógico foi realizada de forma a contemplar os objetivos gerais e específicos esperados, bem como dos perfis profissionais desejados.

Como objetivo geral do curso, busca-se a formação de um profissional com competências e habilidades para: 
a) participar ativamente em todos os âmbitos de atenção à saúde com ações de prevenção, promoção, proteção e reabilitação individual e coletiva;

b) assegurar que sua prática seja realizada de forma integrada e contínua com as instâncias do SUS;

c) pensar criticamente, analisar os problemas da sociedade e procurar soluções para estes;

d) realizar seus serviços dentro dos mais altos padrões de qualidade e dos princípios da ética, tendo em conta que a responsabilidade da atenção à saúde não se encerra no ato técnico, mas sim com a resolução do problema de saúde;

e) tomar decisões visando ao uso apropriado (baseado na utilização eficaz e no custo-efetividade) da força de trabalho, de medicamentos, de equipamentos, de procedimentos e de práticas;

f) avaliar, sistematizar e decidir as condutas mais adequadas, baseadas em evidências;

g) conhecer métodos e técnicas de investigação e elaboração de trabalhos acadêmicos e científicos; dentre outros relacionados à liderança, gestão e educação permanente.

Sua atuação dar-se-á numa perspectiva generalista, humanista, crítica e reflexiva, para atuar com senso de responsabilidade social e compromisso com a cidadania, nos diferentes cenários das práticas de intervenção fisioterapêutica em saúde no âmbito local e regional, no contexto do Sistema Único de Saúde e na perspectiva da promoção da saúde $(7,11,15)$.

Como objetivo específico do curso, busca-se a formação de um profissional com competências e habilidades para:

a) atuar multiprofissionalmente com ações interdisciplinares, com extrema produtividade na promoção da saúde baseada em evidências;

b) garantir a integralidade da assistência, entendida como um conjunto articulado e contínuo das ações e serviços preventivos, curativos, individuais e coletivos que promovam a funcionalidade humana em todos os níveis de complexidade do SUS;

c) contribuir para a manutenção da saúde, bem-estar e qualidade de vida das pessoas, famílias e comunidade, considerando suas circunstâncias éticas, políticas, sociais, econômicas, ambientais e biológicas;

d) realizar consultas, avaliações e reavaliações dos parâmetros relacionados à funcionalidade humana, solicitando, executando e interpretando exames propedêuticos e complementares que permitam elaborar um diagnósticos físico-funcional, para eleger e quantificar as intervenções e condutas fisioterapêuticas;

e) desempenhar atividades de planejamento, organização e gestão de serviços de saúde públicos ou privados, além de assessorar, prestar consultorias e auditorias no âmbito de sua competência profissional;

f) emitir laudos, pareceres, atestados e relatórios;

g) prestar esclarecimento, dirimir dúvidas e orientar o indivíduo e os seus familiares sobre o processo terapêutico;

h) manter a confidencialidade das informações, na interação com outros profissionais de saúde e o público em geral;

i) encaminhar o paciente, quando necessário, a outros profissionais, relacionando e estabelecendo a cooperação com os demais membros da equipe de saúde;

j) manter controle sobre à eficácia dos recursos tecnológicos pertinentes à atuação fisioterapêutica, garantindo sua qualidade e segurança.

Atualmente, o fisioterapeuta é reconhecido de fato como membro da equipe de saúde, com formação específica para desenvolver ações que vão da prevenção ao processo de reabilitação, utilizando programas de orientações e promoção à saúde, além dos agentes característicos da sua atuação (movimento, hidroterapia, termoterapia, eletroterapia e outros).

Deve ser preparado para formular, implantar, organizar, monitorar e avaliar políticas, planos, programas, projetos e serviços de saúde no contexto do SUS. Deve estar comprometido com a ética, valorização e 
defesa da vida, preservação do meio ambiente e cidadania, no atendimento às necessidades sociais em saúde. É, portanto, o trabalhador da saúde em todas as suas dimensões, podendo desempenhar funções nos subsistemas privados de atenção à saúde (13).

$\mathrm{Na}$ perspectiva da responsabilidade e do compromisso social do fisioterapeuta em formação, o curso propõe que este será capaz de:

a) problematizar as situações de saúde em âmbitos local, regional e nacional;

b) reconhecer a transversalidade do saber em saúde;

c) valorizar o aporte de outros campos de saber para a produção de conhecimento no campo da saúde;

d) desenvolver o compromisso com a defesa, implantação, estruturação e organização do Sistema Público de Saúde.

Quanto ao perfil profissional, o fisioterapeuta será capaz de:

a) atuar em todos os níveis de complexidade da atenção a saúde, com conhecimentos acerca das distintas esferas e formas de intervenção fisioterapêutica;

b) ter como objeto de estudo o movimento humano em todas as suas formas de expressão e potencialidades que o permita ampliar a visão de saúde por meio de modelos de funcionalidade, incapacidade e saúde;

c) respeitar as diversidades socioculturais das populações e agir orientado por princípios éticos e humanistas;

d) ter perspectiva de promoção da saúde e atuar voltado às potencialidades de saúde de sujeitos e coletividades.

O enfoque metodológico valoriza os princípios da aprendizagem significativa, que tem na intervenção pedagógica a finalidade de proporcionar ao discente a base necessária para compreender como e porque se relacionam os novos acontecimentos com os que ele já possui, e transmitir-lhe o suporte afetivo que possibilite utilizar estes novos conhecimentos em diferentes contextos. A aprendizagem significativa refere-se ao vínculo entre o novo material de aprendizagem e os conhecimentos prévios dos discentes e à capacidade do discente estabelecer as relações do novo com os seus conhecimentos prévios (20).

Outro traço importante desta proposta é a utilização de metodologias de ensino-aprendizagem que possibilitem aos estudantes ocupar o lugar de sujeitos na construção do conhecimento, tendo o professor como facilitador e orientador desse processo. Assim, os conteúdos são organizados de forma que facilitem a interdisciplinaridade e integralização dos conhecimentos. A integração do ciclo básico com o profissional ocorrerá ao longo de todo o curso, por meio das atividades desenvolvidas nos diferentes cenários das práticas envolvendo o ensino, pesquisa e extensão. A articulação com a Secretaria de Estado da Saúde do Distrito Federal e com a comunidade ocupa um papel fundamental na concretização desta proposta pedagógica e na formação dos futuros profissionais.

Nesse enfoque, os conteúdos são entendidos como fatos, conceitos, princípios, procedimentos, normas e valores, possibilitando, assim, o desenvolvimento de habilidades, para o saber pensar e o aprender a aprender. Desta forma, busca-se o desenvolvimento de habilidades para os estudos autodirigidos, a avaliação crítica das intervenções de saúde e na resolução de problemas, articulando as dimensões individuais e coletivas inseridas no contexto, possibilitando a construção de competências, entendidas nesta perspectiva como um conjunto de saberes (conhecimentos), saber-fazer (práticas), saber-ser (atitudes), saber-agir (mobilização de todos os aspectos para um fazer mais adequado), com as capacidades e habilidades, desenvolvidas por meio da integração das realidades do trabalho e da educação $(8,21)$.

Entende-se, no entanto, que o método de ensino-aprendizagem não deve ser único; ele deve perpassar vários métodos. As estratégias que possibilitam a integração do ensino, da pesquisa e da extensão têm caráter central, refletida nas atividades de campo e de pesquisas, voltada para as necessidades da realidade local; na busca de parcerias com a comunidade, estimuladas especialmente pelo envolvimento 
dos serviços no processo de formação, a exemplo da participação dos profissionais da rede de saúde, no papel de preceptores.

Por fim, cabe ressaltar a permanente articulação entre o ensino e os serviços de saúde, a partir dos primeiros semestres, garantindo-se que os estudantes possam transitar e desenvolver experiências nos três níveis de complexidade do Sistema de Atenção à Saúde, mediante relações de preceptoria, tutoria e monitoria.

\section{Considerações finais}

A diversificação das práticas no contexto do SUS inclui uma perspectiva de transformação social e econômica da população no cenário social e político do Distrito Federal. Neste contexto, prioriza-se a formação do profissional fisioterapeuta generalista, reflexivo e crítico, envolvido desde o princípio com o atendimento à comunidade nos diferentes níveis de atenção, enfocando os aspectos da funcionalidade humana.

\section{Agradecimentos}

Agradecemos a todo o Colegiado do Curso de Fisioterapia da Faculdade de Ceilândia (UnB), que muito contribuiu e tem contribuído para a construção do projeto pedagógico e para a proposta de formação do fisioterapeuta no Distrito Federal.

\section{Referências}

1. Rebelatto JR, Botomé SP. Fisioterapia no Brasil: fundamentos para uma ação preventiva e perspectivas profissionais. 2a ed. São Paulo: Manole; 1999.

2. Brasil. Decreto Lei n. 938, de 13 de outubro de 1969. Prevê sobre as profissões de fisioterapeuta e terapeuta ocupacional, e dá outras providências. Diário Oficial [da] República Federativa do Brasil, Brasília, DF, 13 out. 1969. Seção1, p. 39-40. [acesso 20 set. 2009]. Disponível em: http://www.portaleducacao.com.br/fisioterapia/ artigos/1763/decreto-lei-n-938-de-13-de-outubro-de-1969

3. Rebelatto JR. Fisioterapia cotidiana: ações profissionais e decorrências para a população. Rev Fisioterapia da Univ. São Paulo. 1998;5(1):36-48.

4. Rouquayrol MZ, Almeida Filho N. Epidemiologia e saúde. 5a ed. Rio de Janeiro: MEDSI;1999.

5. Lucas, RWC. Fisioterapia: denominação inadequada para uma atuação profissional moderna. Conhecimento Interativo. 2005;1(1):89-97.

6. Organização Mundial de Saúde (OMS) / Organização Panamericana de Saúde (OPAS). CIF classificação internacional de funcionalidade, incapacidade e saúde. São Paulo: Universidade de São Paulo; 2003.

7. Brasil. Resolução CNE/CES n. 4, 19 out. 2002. Institui Diretrizes Curriculares Nacionais do Curso de Graduação em Fisioterapia. [acesso 25 set. 2009]. Disponível em: http://portal.mec.gov.br/cne/arquivos/pdf/CES042002.pdf

8. Gallo, DLL. A fisioterapia no programa de saúde da família: percepções em relação à atuação profissional e à formação universitária (dissertação). Londrina: Universidade Estadual de Londrina, 2005.

9. Ross, EC, Anderson, EZ. The evolution of a physical therapy research curriculum: integrating evidence-based practice and clinical decision making. J Phys Ther Education. 2004;18(3):52-7.

10. Village,D. Qualities of effective servicelearning in physical therapist education.J Phys TherEducation. 2006;20(3):8-17. 
11. Dornelas de Andrade, A, Dean E. Direcionando a prática da fisioterapia com as principais prioridades de Saúde no Brasil: uma "chamada para ação" no século XXI. Rev Bras Fisioter. 2008,12(4):260-7.

12. Moura Filho, AG. Brasil: a fisioterapia e o tempo. Salvador: [s.n.]; 1999.

13. Ceccim RB, Feuerwerker LC, Macruz. Mudança na graduação das profissões de saúde sob o eixo da integralidade. Cad. Saúde Pública. 2004;20(5):1400-10.

14. Duijn AJV, Bevins SI. Clinical performances of physical therapist students in problem-based, mixed-model, and traditional curricula. J Phys Ther Education. 2005;19(2):15-21.

15. Cavalcanti RA. Andragogia: aprendizagem nos adultos. Rev Clin Cirurg. 1999;6(4):1-6.

16. McCallum C. A process of curriculum development: meeting the needs of a community and a professional physical therapist education program. J Phys Ther Education. 2008;22(2):18-28.

17. Weddle ML, Sellheim DO. An integrative curriculum model preparing physical therapists for vision 2020 practice. J Phys The Education. 2008;23(1):12-20.

18. Farias N, Buchalla CM. The international classification of functioning, disability and health: concepts, uses and perspectives. Rev Bras Epidemiol. 2005;8(2):187-93.

19. Gupta J. A model for interdisciplinary service-learning experience for social change. J Phys Ther Education. 2006;20(3):55-60.

20. Filippin LI, Wagner MB. Fisioterapia baseada em evidência: uma nova perspectiva. Rev Bras Fisioter. 2008;12(5):432-3.

21. Romani WA, Holbert RL. A wellness service-learning project improves the perception of professional empowerment in physical therapist students. J Phys Ther Education. 2007;21(2):73-8.

Recebido: 02/09/2009

Received: 09/02/2009

Aprovado: 23/03/2010

Approved: 03/23/2010

Revisado: 13/05/2010

Reviewed: 05/13/2010 